\title{
Passive seismic experiment and ScS wave splitting in the southwestern subbasin of South China Sea
}

\author{
RUAN AiGuo ${ }^{1}$, LI JiaBiao $^{1 *}$, LEE ChaoShing $^{2}$, QIU XueLin $^{3} \&$ PAN ShaoJun ${ }^{1}$ \\ ${ }^{1}$ The Second Institute of Oceanography, State Oceanic Administration, Hangzhou 310012, China; \\ ${ }^{2}$ Institute of Applied Geosciences, Taiwan Ocean University, Keelung 20224, China; \\ ${ }^{3}$ South China Sea Institute of Oceanology, Chinese Academy of Sciences, Guangzhou 510301, China
}

Received December 22, 2011; accepted February 29, 2012; published online May 6, 2012

\begin{abstract}
The seismic experiment of 3D array of OBS in the southwestern sub-basin of the South China Sea (SCS) is briefly introduced in this paper. The data analysis of broadband OBS shows that totally 93 earthquakes with magnitude of Ms 6.0-6.9 and 10 earthquakes with magnitude above $M \mathrm{~s} 7.0$ were recorded in high quality during this experiment, especially the catastrophic earthquake Ms 9.0 occurred in the east sea area of Japan on March 11, 2011. The anisotropy parameters inversion of ScS wave of four events above $M \mathrm{~s} 7.0$ indicates that the fast direction of shear wave is $\mathrm{N} 58^{\circ} \mathrm{E}$ parallel to the ceased spreading ridge of the southwestern sub-basin of SCS (the slow direction is $\mathrm{S} 35^{\circ} \mathrm{E}$ perpendicular to the spreading ridge), which means the spreading ridge is under compressing stress at present and the cessation of seafloor spreading is related to such stress field as well.
\end{abstract}

broadband OBS, southwestern subbasin of SCS, passive seismic experiment, anisotropy, spreading ridge

Citation: Ruan A G, Li J B, Lee C S, et al. Passive seismic experiment and ScS wave splitting in the southwestern subbasin of South China Sea. Chin Sci Bull, 2012, 57: 3381-3390, doi: 10.1007/s11434-012-5132-0

In early, the crust and mantle structure of the South China Sea (SCS) was studied mainly by using gravity data [1-3] and sonobuoy data [4-6], and the lithosphere structure was studied mainly by using surface waves data of natural earthquakes [7-9]. More accurate and reliable results of crustal structure were from active wide-angle seismic experiments but only in the northern margin of SCS [10-17]. In recent years several ocean bottom seismometer (OBS) experiments in the southern margin of SCS have been carried out that greatly improved the active seismic data distribution in SCS (Figure 1). In the area of SCS, except Manila trench [18], earthquake occurrence frequency is very low and no seismic station in the sea basin. Therefore the study of more accurate and fine crustal structure in SCS by using passive seismic data needs works in two aspects: fully use of available seismic stations on coast and islands and conducting international or regional cooperation to build

*Corresponding author (email: jbli@ sio.org.cn) some new stations for seismic ray covering the whole area of SCS, and deployment some OBS on seafloor to accumulate data with time.

From December 2010 to March 2011, an active seismic experiment of 3D array of OBS was carried out on the ceased spreading ridge of the southwestern sub-basin of SCS (Figure 1). This OBS array is located on the Zhongnan-Changlong seamount chain and consists of 40 OBS among which 8 OBS are broadband I-4C type made in China but one lost (station OBS9). The experiment was lasted for nearly four months due to bad weather. On the other side, broadband OBS recorded many earthquakes including the catastrophic earthquake Ms 9.0 occurring on March 11, 2011 in the east sea of Japan. This paper estimated these natural earthquake data recorded during our 3D OBS experiment in the southwestern subbasin of SCS and calculated the lithosphere anisotropy beneath OBS array by using $\mathrm{ScS}$ wave splitting method.

The anisotropy inversion of lithosphere is based on nu- 


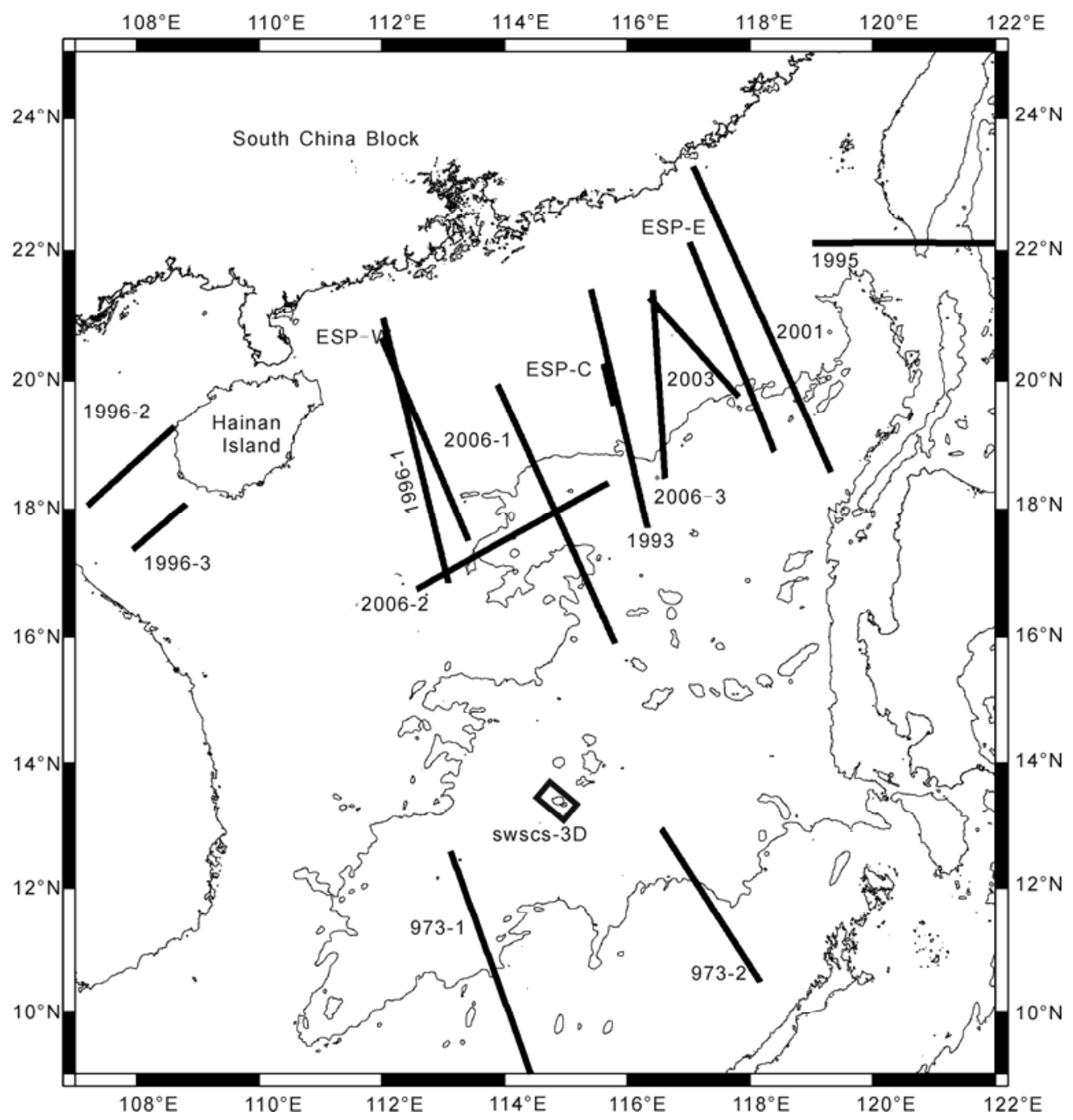

Figure 1 The location of the seismic experiment of 3D OBS array in the southwestern subbasin of SCS. The small square is the OBS array and the solid line is previous OBS experiments.

merous practice and theoretical studies. Since the creative work of Hess in 1966 [19], various seismic observations indicated the presence of anisotropy in the lithosphere or upper mantle and suggested the anisotropy was due to the preferential orientation of mineral lattice resulted by tectonic strains [20]. Although there are some diversities among various hypotheses about the anisotropy mechanism, in general, it is believed that the anisotropy characteristics of the earth reflect the moving direction of deep media of lithosphere. The research approaches of lithosphere anisotropy could be classified two types, one is based on the ray travel time variation passing various azimuths, the other is based on shear velocity variation (splitting) between SH component and SV component due to their different polarizations, such as SKS wave through the inner core of the earth and $\mathrm{ScS}$ wave reflected from the discontinuity between mantle and out core of the earth. Many achievements have been reached in seismic anisotropy research during several decades development but mostly in continent [21]. For marine area seismic surface wave or earthquake records in island station were used. The use of broadband OBS is very fewer, especially in China. In this paper only ScS wave data is used since no suitable SKS wave data in our experiment.

\section{Tectonic setting and OBS array}

The southwestern sub-basin of SCS is a small sea basin formed by seafloor spreading and looks as a trumpet northeast forward opening. Its center is Zhongnan-Changlong seamount chain and a lift valley with thick sediments, showing its characteristic of slow spreading. The magnetic anomaly indicated its seafloor spreading time was 23-16 Ma [22,23], and the seamount distribution indicated some magmatism after the cessation of seafloor spreading. The chronology analysis of basalt rock dredged from the seamount indicated the age of rock was 14-3.5 Ma [24,25], implying magmatism was not simultaneously stopped after the cessation of seafloor spreading. Therefore, it is reasonable to expect the existence of residual magma chamber or 
some special structure beneath the ceased ridge of the southwestern sub-basin of SCS.

This active seismic experiment of 3D array of OBS was originally designed to get fine structure of the ceased spreading ridge, rift valley, oceanic crust and the residual magma chamber in particular. For this purpose, the OBS stations were densely deployed in interval of $10-15 \mathrm{~km}$ (Figure 2), five broadband OBS in the northwest of valley and three broadband OBS in the southeast of the valley, their coordinates are listed in Table 1.

\section{Equipment and data acquisition}

The broadband OBS of I-4C type adopted some new techniques such as rechargeable lithium battery, blue tooth for data input or output and GPS communication method, etc. to follow the technology development in the world. The main part of the OBS includes a status controllable geophone of three components with band of $60 \mathrm{~s}-50 \mathrm{~Hz}$ equipped in a container, a hydrophone and a compass for the azimuths measurement of horizontal components. On the input interface of the preamplifier circuit of recorder a low-pass anti-alias LC filter of first order without source is added and the amplifier circuit of the equipment consists of very low noise, high accuracy and double calculation amplifier for high ability of anti-jamming. The A/D converter adopts $\Sigma-\Delta$ fourth order delta modulator with dynamic rage $>120 \mathrm{~dB}$, data capacity $16 \mathrm{G}$, total power consumption $<0.3 \mathrm{~W}$ and inner clock accuracy better than $5 \times 10^{-8} \mathrm{~s}$. The data sampling interval of this experiment was $8 \mathrm{~ms}$. Figure 3 shows the air gun records of OBS06 station with water depth of $3580 \mathrm{~m}$, air gun volume of 6000 cubic inch and pressure of 120 $\mathrm{kg} / \mathrm{cm}^{2}$. It is showed that, except seismic signals, the water waves were also recorded that could be classified to two kinds according to their frequency, one is a long wave with period of $50 \mathrm{~s}$, the other is a short wave with higher frequency that maybe due to the fact that the height of OBS bottom relative to seafloor is too larger to resist the micro-vibration caused water wave.

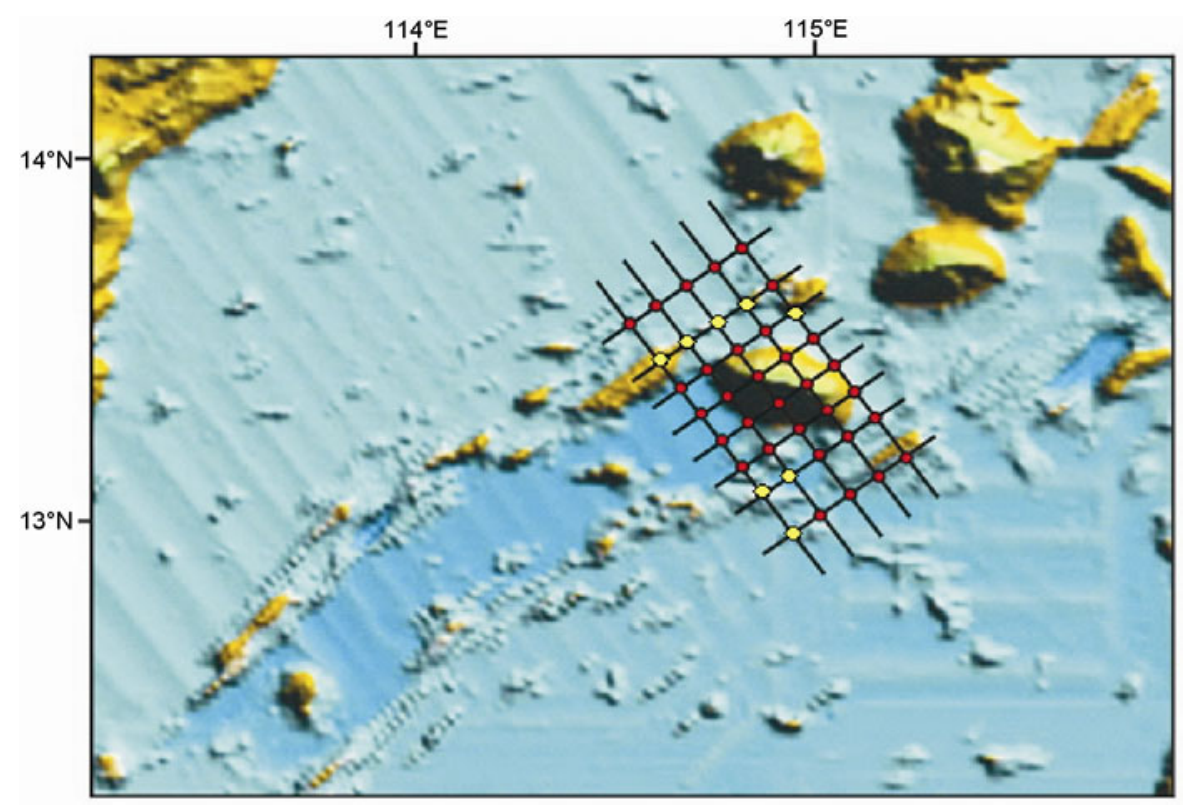

Figure 2 The OBS deployment on the seafloor. The OBS in yellow color are broadband OBS, OBS09 (the fourth from northwest to southeast in the second row) was lost.

Table 1 Station parameter and recording length ${ }^{\text {a) }}$

\begin{tabular}{|c|c|c|c|c|c|c|c|}
\hline Station & OBS06 & OBS07 & OBS08 & OBS011 & OBS34 & OBS35 & OBS36 \\
\hline Lat. $\left({ }^{\circ}\right)$ & 13.44 & 13.49 & 13.54 & 13.57 & 13.12 & 13.06 & 12.95 \\
\hline Lon. $\left({ }^{\circ}\right)$ & 114.60 & 114.69 & 114.76 & 114.97 & 114.95 & 114.87 & 114.95 \\
\hline$\phi\left(^{\circ}\right)$ & 218.50 & 104.50 & 208.50 & 286.00 & 128.00 & 31.50 & 170.50 \\
\hline Starting date & $2010-12-10$ & $2010-12-10$ & $2010-12-10$ & $2010-12-10$ & $2010-12-10$ & $2010-12-10$ & $2010-12-10$ \\
\hline Starting time & 13:02:15 & $13: 12: 38$ & $12: 04: 13$ & 11:02:20 & $12: 49: 47$ & 12:38:01 & 13:27:11 \\
\hline Stopping date & $2011-03-20$ & $2011-02-27$ & $2011-01-10$ & $2010-12-24$ & 2011-02-05 & 2011-03-14 & $2011-02-05$ \\
\hline
\end{tabular}

a) $\phi$ is the compass parameter, the azimuth of the first horizontal component is $X=\phi-60^{\circ}$, for the second component $Y=\phi+30^{\circ}$. The time is in GMT. 

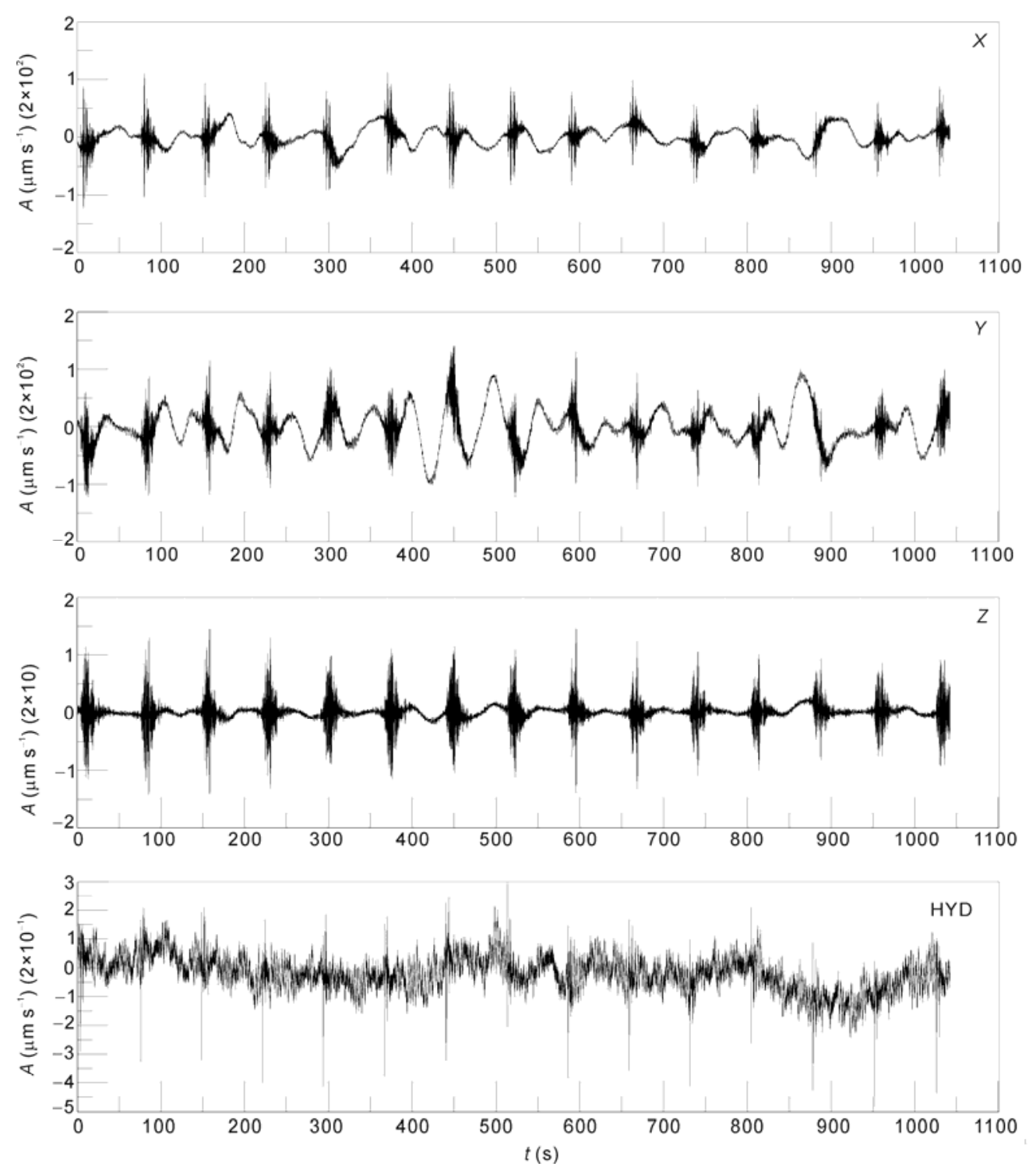

Figure 3 The signal of the airgun recorded by broadband OBS.

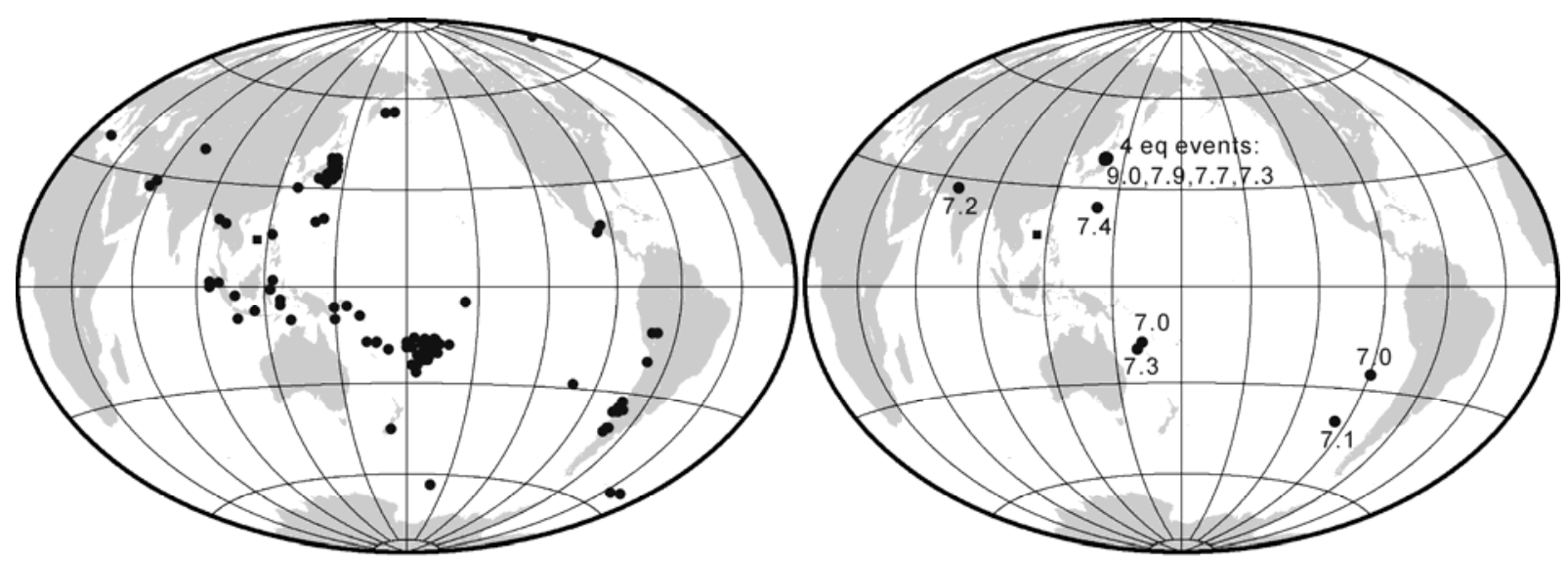

(a)

(b)

Figure 4 Distribution of OBS array and earthquakes. (a) The solid square is OBS array, solid circles are epicenters of earthquake Ms 6.0-6.9; (b) earthquake $\geqslant M \mathrm{~s}$ 7.0. According to data quality, four earthquakes $\geqslant M \mathrm{~s} 7.0$ and one earthquake of $M \mathrm{~s} 6.5$ with much deeper focus are selected for inversion of ScS wave splitting. 
The start recording time of the broadband OBS is December 10, 2010 and the ending time is March 23, 2011. During this period 93 earthquakes of Ms 6.0-6.9 (Figure 4(a)) and 10 earthquakes of $M \mathrm{~s} 7.0$ or greater (Figure 4(b), Table 2) were recorded. The distance and back-azimuth of the 10 earthquake of $M \mathrm{~s} 7.0$ or greater are listed in Table 3. The most important thing is that two broadband OBS rec- orded the catastrophic earthquake Ms 9.0 occurred on March 11, 2011 in the east sea of Japan, and Figure 5 shows the records of OBS06 station with distance $34.93^{\circ}$, back azimuth $39.82^{\circ}$ and first arrival time of $P$ wave 6 63.95" and

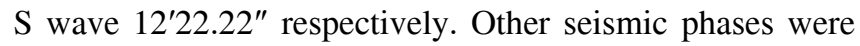
also distinguished such as $\mathrm{ScS}$. These high quality data will play an important role in future studies.
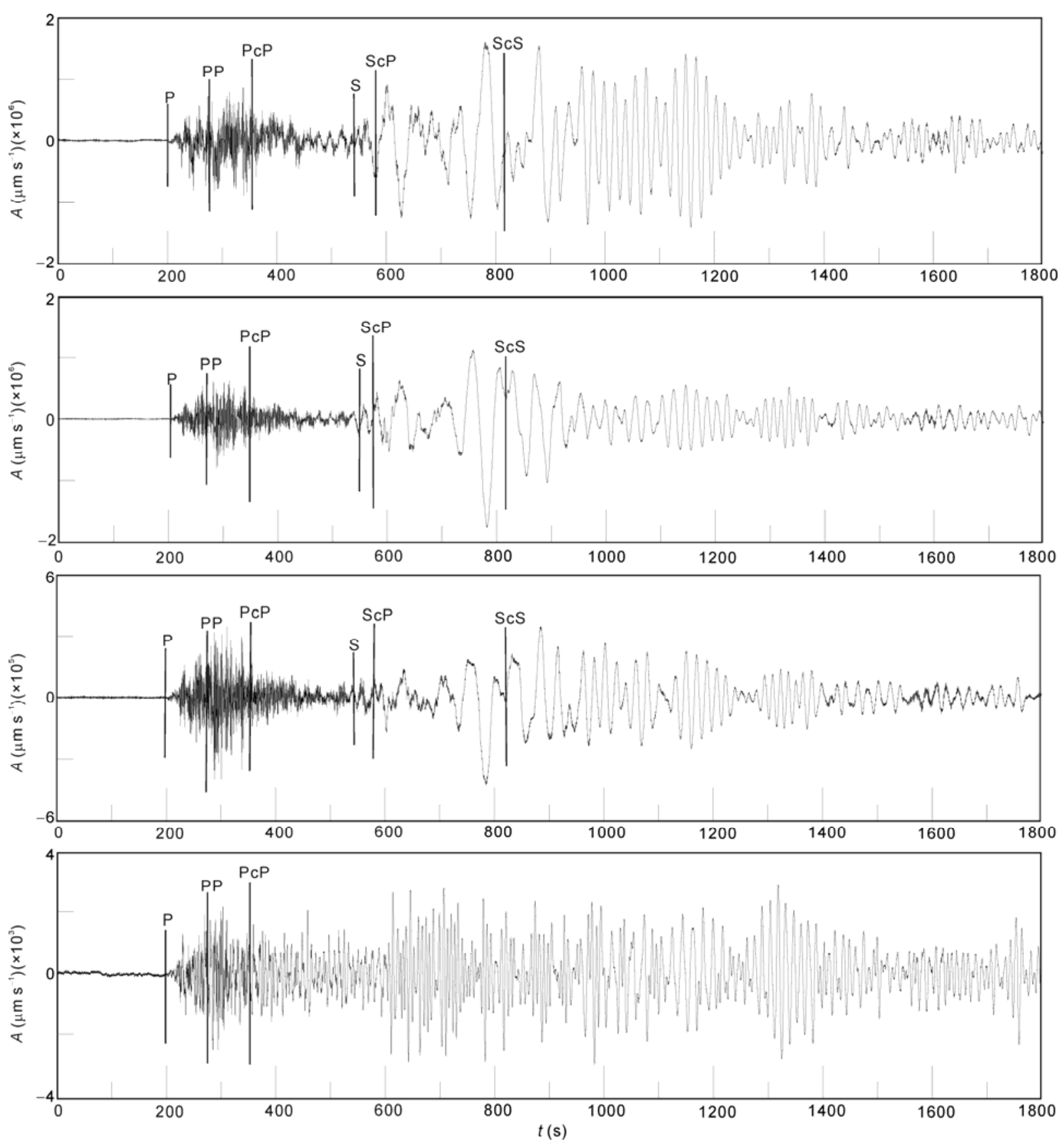

Figure 5 The OBS06 station record of the catastrophe earthquake Ms 9.0 occurred on March 11, 2011 in the east sea of Japan. From top to bottom are horizontal, vertical and hydrophone components respectively. 
Table 2 Earthquake catalogue ( $\geqslant M$ s7.0) from Dec. 2010 to Mar. 2011 ${ }^{\text {a) }}$

\begin{tabular}{ccccccc}
\hline Event & Date & Time $(\mathrm{GMT})$ & Lat. $\left({ }^{\circ}\right)$ & Lon. $\left({ }^{\circ}\right)$ & Depth $(\mathrm{km})$ & Magnitude $(M \mathrm{~s})$ \\
\hline 1 & $2010-12-21$ & $17: 19: 40.66$ & 26.90 & 143.70 & 14 & 7.4 \\
2 & $2010-12-25$ & $13: 16: 37.00$ & -19.70 & 167.95 & 16 & 7.3 \\
3 & $2011-01-01$ & $09: 56: 58.06$ & -26.79 & -63.09 & 576 & 7.0 \\
4 & $2011-01-02$ & $20: 20: 17.69$ & -38.37 & -73.35 & 24 & 7.1 \\
5 & $2011-01-13$ & $16: 16: 41.54$ & -20.63 & 168.47 & 9 & 7.0 \\
6 & $2011-01-18$ & $20: 23: 23.51$ & 28.78 & 63.94 & 68 & 7.2 \\
7 & $2011-03-09$ & $02: 45: 20.33$ & 38.44 & 142.84 & 32 & 7.3 \\
8 & $2011-03-11$ & $05: 46: 24.12$ & 38.30 & 142.37 & 29 & 9.0 \\
9 & $2011-03-11$ & $06: 15: 40.92$ & 36.27 & 141.11 & 48 & 7.9 \\
10 & $2011-03-11$ & $06: 25: 50.30$ & 38.06 & 144.59 & 18 & 7.7 \\
\hline
\end{tabular}

a) This catalogue was published by USGS.

Table 3 The distance and back azimuth of earthquakes ( $\geqslant M \mathrm{~s} 7.0)$ to stations

\begin{tabular}{|c|c|c|c|c|c|c|c|c|c|c|c|}
\hline & Event & 1 & 2 & 3 & 4 & 5 & 6 & 7 & 8 & 9 & 10 \\
\hline \multirow{2}{*}{ OBS06 } & Dis. $\left({ }^{\circ}\right)$ & 30.33 & 61.98 & 166.54 & 154.18 & 62.84 & 49.31 & 35.31 & 34.93 & 32.90 & 36.20 \\
\hline & $\operatorname{BAZ}\left({ }^{\circ}\right)$ & 59.32 & 121.09 & 188.90 & 165.54 & 121.76 & 296.44 & 40.01 & 39.82 & 41.61 & 41.92 \\
\hline \multirow{2}{*}{ OBS07 } & Dis. $\left({ }^{\circ}\right)$ & 30.23 & 61.93 & 166.60 & 154.21 & 62.79 & 49.36 & 35.21 & 34.83 & 32.81 & 36.10 \\
\hline & $\operatorname{BAZ}\left(^{\circ}\right)$ & 59.34 & 121.16 & 188.60 & 165.37 & 121.83 & 296.38 & 39.98 & 39.78 & 41.58 & 41.90 \\
\hline \multirow{2}{*}{ OBS08 } & Dis. $\left({ }^{\circ}\right)$ & 30.14 & 61.89 & 166.66 & 154.24 & 62.76 & 49.40 & 35.13 & 34.75 & 32.72 & 36.02 \\
\hline & $\operatorname{BAZ}\left({ }^{\circ}\right)$ & 59.36 & 121.22 & 188.34 & 165.21 & 121.88 & 296.34 & 39.97 & 39.77 & 41.57 & 41.88 \\
\hline \multirow{2}{*}{ OBS11 } & Dis. $\left({ }^{\circ}\right)$ & 29.96 & 61.74 & 166.72 & 154.22 & 62.60 & 49.57 & 34.98 & 34.60 & 32.57 & 35.86 \\
\hline & $\operatorname{BAZ}\left({ }^{\circ}\right)$ & 59.28 & 121.34 & 187.57 & 164.84 & 122.00 & 296.29 & 39.82 & 39.62 & 41.41 & 41.75 \\
\hline \multirow{2}{*}{ OBS34 } & Dis. $\left({ }^{\circ}\right)$ & 30.20 & 61.52 & 166.27 & 153.79 & 62.38 & 49.75 & 35.33 & 34.96 & 32.92 & 36.21 \\
\hline & $\operatorname{BAZ}\left({ }^{\circ}\right)$ & 58.63 & 121.12 & 187.4 & 165.11 & 121.79 & 296.63 & 39.43 & 39.23 & 40.97 & 41.36 \\
\hline \multirow{2}{*}{ OBS35 } & Dis. $\left({ }^{\circ}\right)$ & 30.29 & 61.56 & 166.21 & 153.76 & 62.42 & 49.71 & 35.42 & 35.05 & 33.00 & 36.30 \\
\hline & $\operatorname{BAZ}\left(^{\circ}\right)$ & 58.60 & 121.06 & 187.66 & 165.27 & 121.73 & 296.68 & 39.45 & 39.25 & 40.99 & 41.37 \\
\hline \multirow{2}{*}{ OBS36 } & Dis. $\left({ }^{\circ}\right)$ & 30.29 & 61.43 & 166.11 & 153.63 & 62.82 & 49.83 & 35.46 & 35.08 & 33.04 & 36.33 \\
\hline & $\operatorname{BAZ}\left({ }^{\circ}\right)$ & 58.39 & 121.05 & 187.31 & 165.20 & 122.16 & 296.75 & 39.29 & 39.08 & 40.80 & 41.21 \\
\hline
\end{tabular}

\section{Shear wave splitting of earthquake with magnitude $M s \geqslant 7.0$}

When seismic waves propagate through anisotropy medium shear wave splitting phenomenon will occur [26,27]. In anisotropy medium different polarized shear waves have different velocities, so the originally combined linear trace of two shear waves is destroyed after spliting through anisotropy medium. If one can properly rotate the split shear wave components and correct the time delay between them, the original two shear waves without splitting can be separated from each other and the linear trace can be recovered. The determined rotation angle is the polarization of fast shear wave that corresponds to the direction of tensile strain or the moving direction of plate or mantle flow and the time delay between the fast and slow waves is related to the anisotropy strength and the effective thickness of anisotropy medium.

During our experiment 10 earthquakes with magnitude $\geqslant$ Ms 7.0 were observed (Table 2) and ScS waves from four of them are selected to calculate anisotropy parameters by rotating correlation function technique [28]. The procedure is to separate two horizontal components by projecting them upon the rotated axes, then make correction of time delay between them and calculate their correlation function. The rotation angle and time delay corresponding to the maximum correlation function are taken as final inversed parameters. Suppose one horizontal component is $\mathrm{NS}(Y)$, another is $\mathrm{EW}(X)$, rotate them clockwise in the range of $0^{\circ}-180^{\circ}$ with interval $\Delta \varphi=2^{\circ}$, that is

$$
\begin{gathered}
S_{1}=Y \cos \varphi+X \sin \varphi, \\
S_{2}=-Y \sin \varphi+X \cos \varphi .
\end{gathered}
$$

For each rotation angle, the time delay $\tau$ is gradually increased in the range of $-2.4-2.4 \mathrm{~s}$ with interval $\Delta \tau=0.8 \mathrm{~s}$. The correlation function is

$$
C(\phi, \tau)=\int_{t_{1}}^{t_{2}} S_{1}(t) S_{2}(t+\tau) \mathrm{d} t,
$$

where $t_{1}$ and $t_{2}$ are start point and end point of data selected. Considering the existence of disturbances, the data should be filtered before inversion.

The inversed shear wave splitting parameters of lithosphere of our experiment is listed in Table 4. Except in some cases that without data or inversion abortive, the most 
solutions are stable, fast direction is $56^{\circ}-60^{\circ}$ and time delay is $0.8-1.04 \mathrm{~s}$. Obviously there are some great diversity cases because of various kinds of disturbances and some uncertain effects. Random disturbances would affect recording quality of data and the locating errors would result in some errors in the calculation of distance and arrival time, e.g. the error in distance and travel time due to earthquake locating will affect the first arrival of ScS. The earthquake itself, including focal depth, focus process, will cause error in anisotropy calculation. The anisotropy effects of split ScS wave is from the medium in the total ray path, so the inversed anisotropy parameters could be affected by the heterogeneity in ray path and surface waves as well. Therefore the estimation or checking of inversed parameters is neces- sary.

The inversed results are assessed from three aspects (taking event 3 of OBS06 station as an example). First, it is easy to see that the original waves of two components are not in agreement due to splitting (Figure 6(a)), while after correction they are in agreement well (Figure 6(c)). Second, it is obvious that after correction the particle trace presents linearly characteristics (Figure 6(d)), in contrast, before correction the trace is much complicated (Figure 6(b)). Third, the parameters (central small circle) directly read from correlation contour (Figure 6(e)) are identical to extremes obtained from inversion. It is similar to the results of Japan east sea earthquake (event 8) of OBS06 and OBS35 (Figures 7 and 8). Furthermore according to Table 4, we take

Table 4 The parameters of ScS wave anisotropy ${ }^{\text {a) }}$

\begin{tabular}{|c|c|c|c|c|c|}
\hline Event & 1 & 2 & 5 & 8 & 11 \\
\hline Parameter & $\phi / \delta t$ & $\phi / \delta t$ & $\phi / \delta t$ & $\phi / \delta t$ & $\phi / \delta t$ \\
\hline OBS06 & $56 / 0.8$ & $40 / 1.04$ & $58 / 0.8$ & $56 / 0.88$ & $56 / 0.8$ \\
\hline OBS07 & $28 / 0.8$ & $58 / 1.04$ & $58 / 0.64$ & no data & $60 / 0.8$ \\
\hline OBS08 & abortive & $62 / 0.96$ & no data & no data & no data \\
\hline OBS11 & $56 / 0.8$ & no data & no data & no data & no data \\
\hline OBS34 & $60 / 1.04$ & $60 / 0.96$ & $40 / 0.96$ & no data & $55 / 0.96$ \\
\hline OBS35 & $76 / 0.8$ & $16 / 0.88$ & no data & 58/0.8 & no data \\
\hline OBS36 & $58 / 1.04$ & $58 / 0.8$ & $58 / 0.96$ & no data & $56 / 0.8$ \\
\hline
\end{tabular}

a) $\phi$ is fast direction (relative to north clockwise) (degree), $\delta t$ is time delay (s).
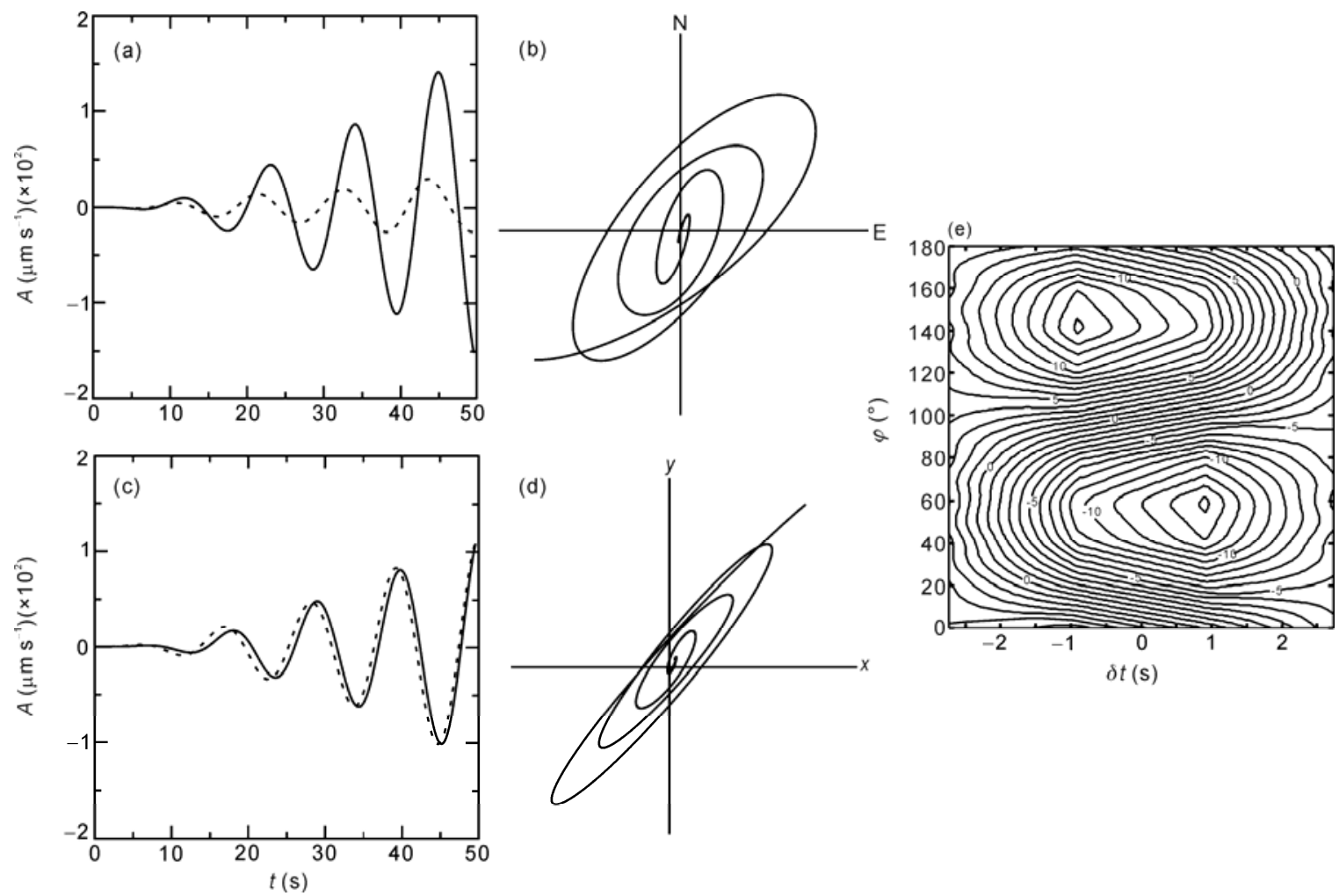

Figure 6 Analysis of inversion result of event 1 of OBS06 station. (a) Horizontal components of ScS before inversion; (b) wave trace of ScS before inversion; (c) horizontal components of ScS after inversion; (d) wave trace of ScS after inversion; (e) contour of correlation function. 

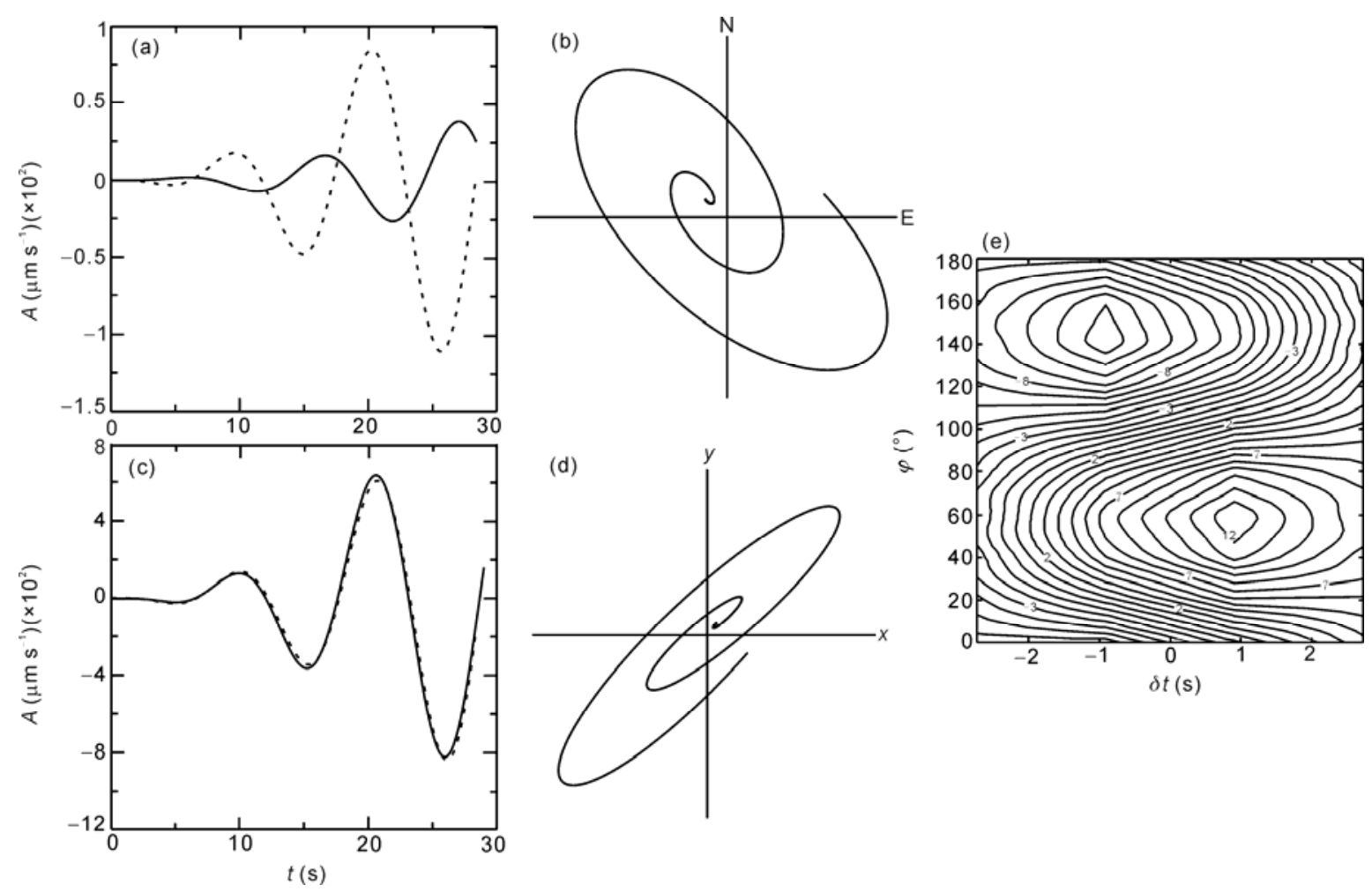

Figure 7 Analysis of inversion result of event 8 of OBS06 station. The others are the same as in Figure 6.
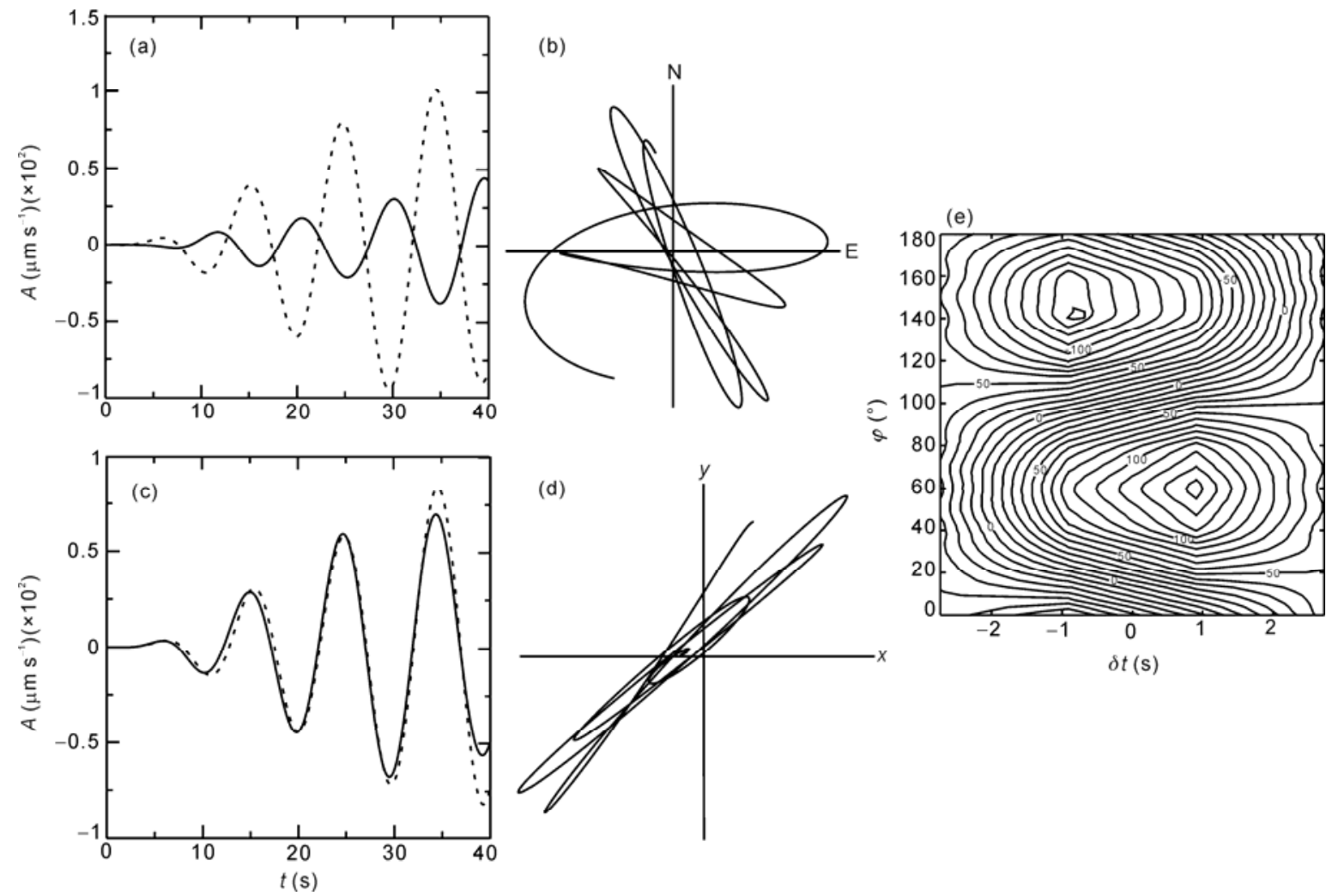

Figure 8 Analysis of inversion result of event 8 of OBS35 station. The others are the same as in Figure 6. 
fast direction of $58^{\circ}$ (from north clockwise) and slow direction of $145^{\circ}$ as mean parameters.

Experiences have shown that the deeper of seismic focus the more reliable of $\mathrm{ScS}$. Therefore, for comparison we selected an earthquake $M$ s 6.5 (event 11 in Table 4), with focus depth $527 \mathrm{~km}$, distance $27.20^{\circ}$ and back-azimuth $56.44^{\circ}$ (relative to OBS06 station), to calculate ScS wave splitting parameters (Table 4, Figure 9) that show much similarity with that of others. According these anisotropy parameter in Table 4, we suggest the fast direction of shear wave is $\mathrm{N} 58^{\circ} \mathrm{E}$ parallel to the ceased spreading ridge of the southwestern sub-basin with time delay of $0.9 \mathrm{~s}$ and the slow direction is $\mathrm{S} 35^{\circ} \mathrm{E}$ (with minus time delay) perpendicular to the spreading ridge.

\section{Discussion and conclusions}

This paper processes and analyzes earthquake records of broadband OBS during a 3D OBS experiment in the southwestern subbasin of SCS. Totally 93 earthquakes $M \mathrm{~s}$ 6.0-6.9 and 10 earthquakes $M \mathrm{~s} 7.0$ or greater were recorded with high quality, especially the catastrophic earthquake $M \mathrm{~s}$ 9.0 occurred on March 11, 2011 in the east sea of Japan. These data will play an important role in future studies.

The stress field of SCS is very complicated due to various dynamic sources in this area. According to the inversion of gravity data, in the southern SCS the lower mantle flowing direction is southeast [29]. The measurement of GPS indicates the South China is moving in southeast direction. The inversion of seismic surface waves indicates the major feature of lithosphere stress of SCS is WE extension in the northwestern area, SN extension in the upper crust and WE extension in the lower crust in the middle area, and WE extension in the southern area [30]. Moreover, numerical simulations suggested some different or conflict ideas about the stress field of SCS. Obviously, shear wave splitting method is a better one for such problems because of its higher accuracy than surface wave and gravity techniques. It is suggested that lithosphere anisotropy is due to the preferential orientation of olivine lattice resulted by strain and the fast direction corresponds to tensile strain caused by the moving of upper mantle or lithosphere.

In this paper the inversed fast direction of $\mathrm{N}^{\circ} 8^{\circ} \mathrm{E}$ parallel to the spreading ridge confirmed a previous deduction that the seafloor spreading of SCS was developed from the central sub-basin to the southwestern subbasin [22], for in such spreading mode the ridge is parallel to the preferential orientation of olivine lattice. The slow direction of $\mathrm{S} 35^{\circ} \mathrm{E}$ perpendicular to the ceased spreading ridge of southwestern subbasin indicates the present stress field of the ridge, reflects the collision situation of the southern margin of SCS and provides some dynamic mechanism explanations for the cessation of the spreading ridge.
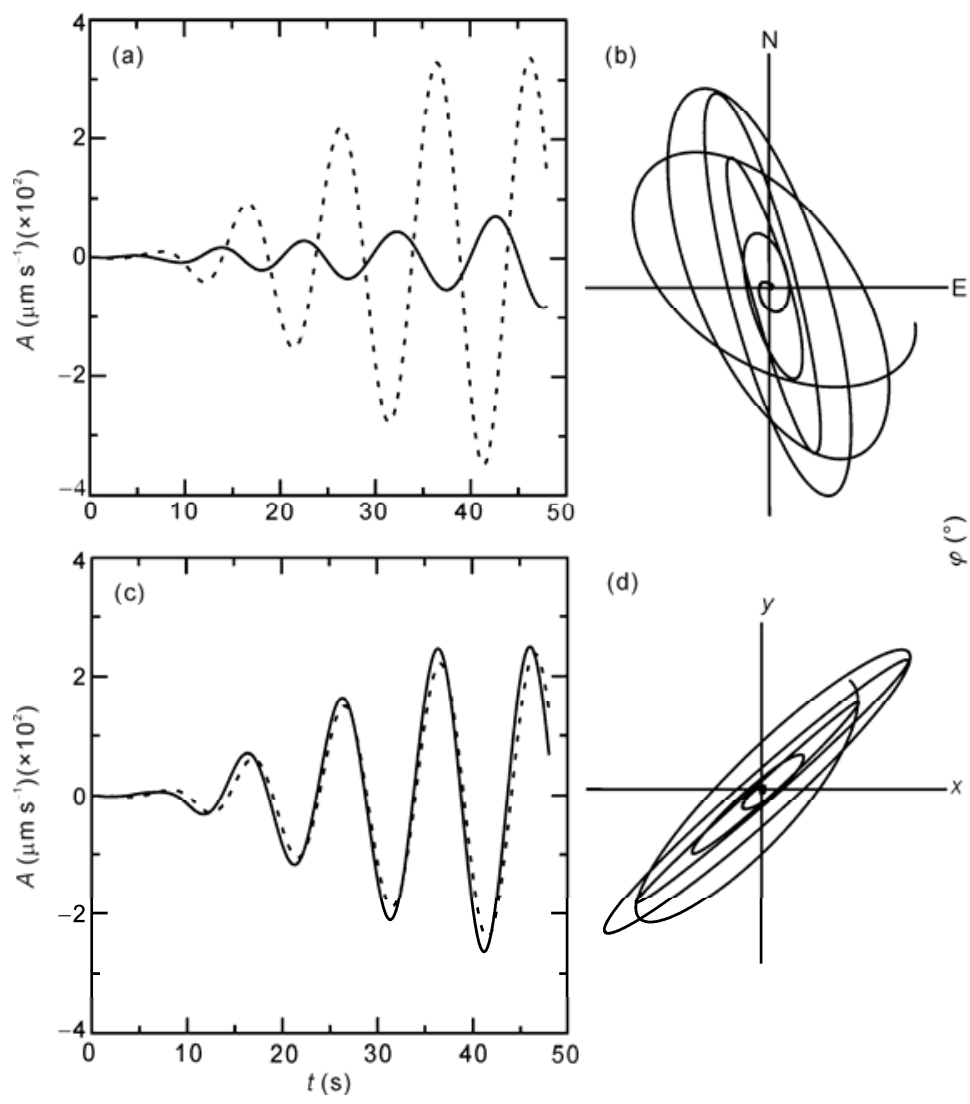

(d)
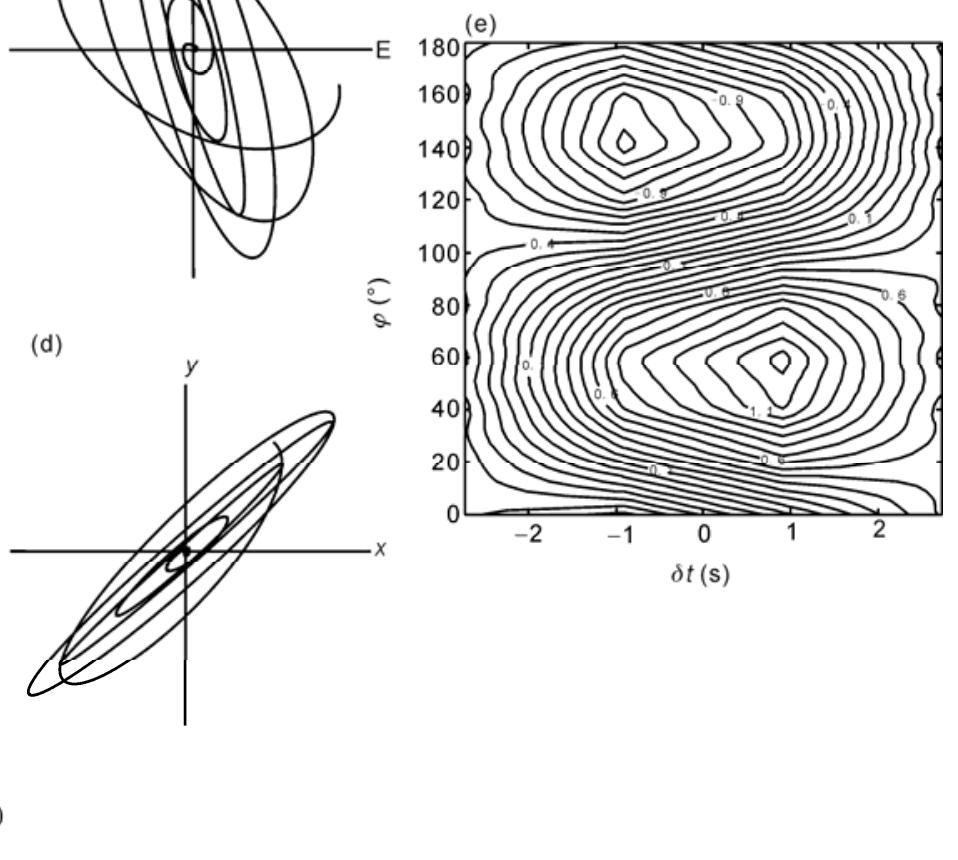

Figure 9 Analysis of inversion result of event $11(M \mathrm{~s} 6.5)$ of OBS06 station. The others are the same as in Figure 6. 
Looking head, we believe these earthquake data can be applied to receiver function method for the shear wave velocity structure of lithosphere beneath the OBS stations. More seismic arrivals will be distinguished form these data and contribute to the seismic data source for the tomography of the whole of SCS.

Thanks to the participators of the OBS experiment, they are Dr. Wu Zhenli, Dr. Dong Chongzhi, Niu Xiongwei and Liu Hongyang from the Second Institute of Oceanography, SOA; Dr. Zhu Junjiang, Huang Haibo and Wei Xiaodong from the South China Sea Institute of Oceanology, CAS; Liang Jinwei, Wang Liangjun, Qiu Maoxiang and Xu Weifeng from the Institute of Applied Geosciences of Taiwan Ocean University. The authors also thank the officers and crew of the research vessels "Fendou Seven" and "Kan 407" of the First Brigade of Marine Geological Survey, Shanghai Marine Oil Bureau and "Shiyan Two" of the South China Sea Institute of Oceanology, CAS. This work was supported by the National Natural Science Foundation of China (91028006, 40876035 and 41106053) and the National Basic Research Program of China (2007CB411701).

1 Liu Z, Wang Q, Yuan H, et al. The Bouguer anomalies and depths of Mohorovicic discontinuity in the South China Sea region. Bull Chin Oceanogr, 1985, 4: 579-590

2 Chen B Y, Lei S M. Map of crustal structures. In: He L S, Chen B Y, eds. Atlas of Geology and Geophysics of South China Sea. Guangzhou: Map Publishing House of Guangdong Province, 1987. 10-12

3 Su D Q, Liu Y L, Chen X. 3D Moho interface of South China Sea. In: Zhang Z J, Gao R, Lu Q T, eds. Deep structure and geodynamic research of continental China. In: Congratulation on the 50 Years Anniversary of Geophysics Carrier of Academician Teng Jiwen. Beijing: Science Press, 2004. 357-365

4 Ludwig W J, Kumar N, Houtx R E. Profiler-Sonobuoy measurements in the South China Sea Basin. J Geophys Res, 1979, 84: 3505-3518

$5 \mathrm{Li} \mathrm{Z} \mathrm{W}$. Discussion on the crust nature of middle and northern part of the South China Sea (in Chinese). Acta Geophys Sin, 1984, 27: 153-165

6 Yao B C, Wang G Y. Crustal structure of South China Sea basin (in Chinese). Sci Chin, 1983, 2: 177-186

7 Yao B C, Wan L, Wu N Y. Cenozoic tectonic evolution and the 3D structure of the ithosphere of the South China Sea (in Chinese). Geol Bull Chin, 2005, 24: 1-8

8 Zhu J S. The structural characteristics of lithosphere in the continent of Eurasia and its marginal seas (in Chinese). Earth Sci Front, 2007, 14: $1-20$

9 Hao T Y, Huang S, Xu Y, et al. Comprehensive geophysical research on the deep structure of Northeastern South China Sea (in Chinese). Acta Geophys Sin, 2008, 51: 1785-1796

10 Nissen S S, Hayes D E, Buhl P, et al. Deep penetration seismic sounding across the northern margin of the South China Sea. J Geophys Res, 1995, 100: 22407-2433

11 Yan P, Zhou D, Liu Z S. A crustal structure profile across the northern continental margin of the South China Sea. Tectonophysics, 2001, 338: $1-21$

12 Qiu X L, Ye S Y, Wu S, et al. Crustal structure across the Xisha Trough, northwestern South China Sea. Tectonophysics, 2001, 341: 179-193

13 Wang T K, Chen M K, Lee C S, et al. Seismic imaging of the transitional crust across the northeastern margin of the South China Sea. Tectonophysics, 2006, 412: 237-254
14 Zhao M H, Qiu X L, Ye C M, et al. An analysis on deep crustal structure along the onshore-offshore seismic profile across the Binhai (littoral) fault zone in NE South China Sea (in Chinese). Acta Geophys Sin, 2004, 47: 845-852

15 Ruan A G, Niu X W, Wu Z L, et al. The $2 \mathrm{D}$ velocity and density structure of the Mesozoic sediments in the Chaoshan Depression (in Chinese). Geol J Chin Univ, 2009, 15: 522-528

16 Wu Z L, Li J B, Ruan A G, et al. Crustal structure of the northwestern sub-basin, South China Sea: Results from a wide-angle seismic experiment. Sci China Earth Sci, 2011, doi: 10.1007/s11430-0114324-9

17 Qiu X L, Chen Y, Zhu R, et al. The application of large volume airgun sources to the onshore-offshore seismic surveys: Implication of the experiment results in northern South China Sea. Chin Sci Bull, 2007, 52: 553-560

18 Li J B, Jin X L, Ruan A G, et al. Identation tectonics in the accretionary wedge of middle Manila Trench (in Chinese). Chin Sci Bull (Chin Ver), 2004, 49: 1279-1288

19 Hess H H. Seismic anisotropy of the uppermost mantle under ocean. Nature, 1964, 203: 629-63

20 Nicolas A, Christensen N I. Formation of anisotropy in upper mantle peridotites - A review in composition, structure and dynamics of the lithosphere. In: Fuches K, Froidevaux C, eds. Asthenosphere System Vol.16. Washington D C: Am Geophys Union, 1987. 111-123

21 Ruan A G, Wang C Y. Overview of research on anisotropy in the upper mantle and the Traim thoughts of researches in the Sichuan and Yunnan regions (in Chinese). Recent Develop World Seismol, 2011, 10: $1-7$

22 Briais A., Patriat P, Tapponnier P. Updated interpretation of magnetic anomalies and seafloor spreading in the South China Sea: Implications for the Tertiary tectonics of Southeast Asia. J Geophy Res, 1993 98: 6299-6328

23 Sun Z, Zhou D, Wu S M, et al. Patterns and dynamics of rifting of passive continental margin from self to slope of the northern South China Sea: Evidence from 3D analogue modeling. J Earth Sci, 2009, 20: $137-146$

24 Yan P, Deng H, Liu H L. The temporal and spatial distribution of volcanism in the South China Sea region. Jas Earth Sci, 2006, 27: 647-659

25 Wang Y J, Han X Q, Luo Z H, et al. Late Miocenic magmatism and evolution of Zhenbei-Huangyan seamount, South China Sea: Evidence from petrochemistry and Chronology. In: Jin X L, Qin Y S, Zhu R X, et al., eds. The Development of Geology and Geophysics in China-Congratulation on the 80 Years Birthday of Academician Liu Guangding (in Chinese). Beijing: Ocean Press, 2008. 658-668

26 Keith C M, Crampin S. Seismic body waves in anisotropic media: Synthetic seismograms. J Geophys Res, 1977, 49: 225-243

27 Stuart C. A review of the effects of anisotropic layering on the propagation of seismic waves. J Geophys Res, 1977, 49: 9-27

28 Bowman J R, Ando M. Shear-wave splitting in the upper mantle wedge above the Tonga subduction zone. J Geophys Res, 1987, 88: 25-41

29 Wang Q L, Liu Z H, Huang C L, et al. Stress field of mantle convection current and continental marginal spread in South China Sea and its vicinity (in Chinese). Tropic Ocenonol, 1987, 6: 19-27

30 Zheng Y J, Huang Z X, Peng Y J. The upper mantle anisotropy and its tectonic implication in the continental margin of China. In: Li J B, Gao S, eds. The Evolution of Sea Basin and Resources Effects of Chinese Margin Sea. 3rd Volume of the Series Researches on the Evolution of Chinese Margin Sea (in Chinese). Beijing: Ceanography Press, 2004. 3-8

Open Access This article is distributed under the terms of the Creative Commons Attribution License which permits any use, distribution, and reproduction in any medium, provided the original author(s) and source are credited. 